LAWRENCE LIVERMORE N A TION A L LABORATORY
Vibration response for a
multilayer cylinder

D. H. Chambers

March 2, 2007 
This document was prepared as an account of work sponsored by an agency of the United States Government. Neither the United States Government nor the University of California nor any of their employees, makes any warranty, express or implied, or assumes any legal liability or responsibility for the accuracy, completeness, or usefulness of any information, apparatus, product, or process disclosed, or represents that its use would not infringe privately owned rights. Reference herein to any specific commercial product, process, or service by trade name, trademark, manufacturer, or otherwise, does not necessarily constitute or imply its endorsement, recommendation, or favoring by the United States Government or the University of California. The views and opinions of authors expressed herein do not necessarily state or reflect those of the United States Government or the University of California, and shall not be used for advertising or product endorsement purposes.

This work was performed under the auspices of the U.S. Department of Energy by University of California, Lawrence Livermore National Laboratory under Contract W-7405-Eng-48. 


\title{
Vibration response for a multilayer cylinder
}

\author{
David H. Chambers
}

February 28, 2007

\section{Analytical solution}

Consider a circular cylinder composed of $N$ concentric layers of materials. Each layer is characterized by its material density $\rho_{n}$, and elastic moduli $\lambda_{n}$ and $\mu_{n}(n=1, \ldots, N)$. The interfaces between layers are specifed by the radii $R_{0}, R_{1}, \ldots, R_{n}$, with $R_{0}$ the inner surface and $R_{N}$ the outer surface of the cylinder. For each layer, the radial displacement field $u_{n}(r, \theta)$ and tangential displacement field $v_{n}(r, \theta)$ can be expressed using two elastic potentials $\phi_{n}(r, \theta)$ and $\psi_{n}(r, \theta)$ :

$$
\begin{aligned}
& u_{n}(r, \theta)=\frac{\partial \phi_{n}}{\partial r}+\frac{1}{r} \frac{\partial \psi_{n}}{\partial \theta}, \\
& v_{n}(r, \theta)=\frac{1}{r} \frac{\partial \phi_{n}}{\partial \theta}-\frac{\partial \psi_{n}}{\partial r} .
\end{aligned}
$$

The radial normal stress $\sigma_{r r}^{(n)}(r, \theta)$ and shear stress $\sigma_{r \theta}^{(n)}(r, \theta)$ are given by

$$
\begin{aligned}
& \sigma_{r r}^{(n)}(r, \theta)=\left(\lambda_{n}+2 \mu_{n}\right) \frac{\partial^{2} \phi_{n}}{\partial r^{2}}+\frac{\lambda_{n}}{r}\left(\frac{\partial \phi_{n}}{\partial r}+\frac{1}{r} \frac{\partial^{2} \phi_{n}}{\partial \theta^{2}}\right)+\frac{2 \mu_{n}}{r}\left(\frac{\partial}{\partial r}-\frac{1}{r}\right) \frac{\partial \psi_{n}}{\partial \theta}, \\
& \sigma_{r \theta}^{(n)}(r, \theta)=\frac{2 m u_{n}}{r}\left(\frac{\partial}{\partial r}-\frac{1}{r}\right) \frac{\partial \phi_{n}}{\partial \theta}+\mu_{n}\left(-\frac{\partial^{2} \psi_{n}}{\partial r^{2}}+\frac{1}{r} \frac{\partial \psi_{n}}{\partial r}+\frac{1}{r^{2}} \frac{\partial^{2} \psi_{n}}{\partial \theta^{2}}\right) .
\end{aligned}
$$

The potentials are solutions to two Helmholtz equations:

$$
\begin{array}{ll}
\nabla^{2} \phi_{n}+k_{L n}^{2} \phi_{n}=0, & k_{L n}^{2}=\frac{\omega^{2}}{c_{L n}^{2}}=\frac{\rho_{n} \omega^{2}}{\lambda_{n}+2 \mu_{n}} ; \\
\nabla^{2} \psi_{n}+k_{T n}^{2} \psi_{n}=0, & k_{T n}^{2}=\frac{\omega^{2}}{c_{T n}^{2}}=\frac{\rho_{n} \omega^{2}}{\mu_{n}} .
\end{array}
$$

For cylindrical geometry, the solutions to the Helmholtz equations can be written as Fourier series in the angular coordinate $\theta$ :

$$
\begin{aligned}
& \phi_{n}(r, \theta)=\sum_{m=0}^{\infty} \varepsilon_{m}(-1)^{m}\left[A_{n}^{(m)} J_{m}\left(k_{L n} r\right)+B_{n}^{(m)} Y_{m}\left(k_{L m} r\right)\right] \cos (m \theta), \\
& \psi_{n}(r, \theta)=\sum_{m=0}^{\infty} \varepsilon_{m}(-1)^{m}\left[D_{n}^{(m)} J_{m}\left(k_{T n} r\right)+E_{n}^{(m)} Y_{m}\left(k_{T n} r\right)\right] \sin (m \theta),
\end{aligned}
$$

where

$$
\varepsilon_{m}=\left\{\begin{array}{ll}
1, & m=0 \\
2, & m \geq 1
\end{array} .\right.
$$

Equations for the coefficients $A_{n}^{(m)}, B_{n}^{(m)}, D_{n}^{(m)}$, and $E_{n}^{(m)}$, are obtained by requiring continuity of displacement and stress across each interface $\left(r=R_{1}, R_{2}, \ldots, R_{N-1}\right)$ and by specifying the tractions on the inner 
$\left(r=R_{0}\right)$ and outer $\left(r=R_{N}\right)$ surfaces. These conditions can be applied independently for each order $m$ in the Fourier series. The final matrix equation for the coefficients of the mth mode is $\mathbf{Z}^{(m)} \cdot \mathbf{C}^{(m)}=\mathbf{b}^{(m)}$, where $\mathbf{C}^{(m)}$ is a vector containing the coefficients, $\mathbf{b}^{(m)}$ is a vector determined from the applied tractions, and $\mathbf{Z}^{(m)}$ is a matrix consisting of Bessel functions evaluated at the interfaces.

The vector $\mathbf{C}^{(m)}$ can be constructed by stacking $N$ vectors $\mathbf{C}_{n}^{(m)}$ :

$$
\mathbf{C}^{(m)}=\left[\begin{array}{c}
\mathbf{C}_{1}^{(m)} \\
\mathbf{C}_{2}^{(m)} \\
\vdots \\
\mathbf{C}_{N}^{(m)}
\end{array}\right], \quad \mathbf{C}_{n}^{(m)}=\left[\begin{array}{c}
A_{n}^{(m)} \\
B_{n}^{(m)} \\
D_{n}^{(m)} \\
E_{n}^{(m)}
\end{array}\right] .
$$

The matrix $\mathbf{Z}^{(m)}$ can be written in terms of the $4 \times 4$ matrices $\mathbf{S}_{n}^{(m)}(r)$ and the $2 \times 4$ matrices $\mathbf{F}_{n}^{(m)}(r)$ :

$$
\mathbf{Z}^{(m)}=\left[\begin{array}{cccc}
\mathbf{F}_{1}^{(m)}\left(R_{0}\right) & \mathbf{0} & \ldots & \mathbf{0} \\
\mathbf{S}_{1}^{(m)}\left(R_{1}\right) & -\mathbf{S}_{2}^{(m)}\left(R_{1}\right) & \ddots & \vdots \\
\mathbf{0} & \ddots & \ddots & \mathbf{0} \\
\vdots & \ddots & \mathbf{S}_{N-1}^{(m)}\left(R_{N-1}\right) & -\mathbf{S}_{N}^{(m)}\left(R_{N-1}\right) \\
\mathbf{0} & \cdots & \mathbf{0} & \mathbf{F}_{N}^{(m)}\left(R_{N}\right)
\end{array}\right] .
$$

The $\mathbf{S}_{n}^{(m)}(r)$ matrices are composed of the $\mathbf{F}_{n}^{(m)}(r)$ matrices and another $2 \times 4$ matrix $\mathbf{G}_{n}^{(m)}(r)$ :

$$
\mathbf{S}_{n}^{(m)}(r)=\left[\begin{array}{l}
\mathbf{F}_{n}^{(m)}(r) \\
\mathbf{G}_{n}^{(m)}(r)
\end{array}\right]
$$

where

$$
\mathbf{G}_{n}^{(m)}(r)=\left[\begin{array}{cccc}
k_{L n} J_{m}^{\prime}\left(k_{L n} r\right) & k_{L n} Y_{m}^{\prime}\left(k_{L n} r\right) & \frac{m}{r} J_{m}\left(k_{T n} r\right) & \frac{m}{r} Y_{m}\left(k_{T n} r\right) \\
\frac{m}{r} J_{m}\left(k_{L n} r\right) & \frac{m}{r} Y_{m}\left(k_{L n} r\right) & k_{T n} J_{m}^{\prime}\left(k_{T n} r\right) & k_{T n} Y_{m}^{\prime}\left(k_{T n} r\right)
\end{array}\right] .
$$

Finally, the $\mathbf{F}_{n}^{(m)}(r)$ matrix is given by

$$
\mathbf{F}_{n}^{(m)}(r)=\left[\begin{array}{cccc}
F_{11 n}^{(m)}\left(k_{L n} r\right) & F_{12 n}^{(m)}\left(k_{L n} r\right) & \frac{2 \rho_{n} \omega c_{T n} m}{r} g_{m}^{(1)}\left(k_{T n} r\right) & \frac{2 \rho_{n} \omega c_{T n} m}{r} g_{m}^{(2)}\left(k_{T n} r\right) \\
\frac{2 \rho_{n} \omega c_{T n}^{2} m}{c_{L n} r} g_{m}^{(1)}\left(k_{L n} r\right) & \frac{2 \rho_{n} \omega c_{T n}^{2} m}{c_{L n} r} g_{m}^{(2)}\left(k_{L n} r\right) & F_{23 n}^{(m)}\left(k_{T n} r\right) & F_{24 n}^{(m)}\left(k_{T n} r\right)
\end{array}\right],
$$

with

$$
\left[F_{11 n}^{(m)}\left(k_{L n} r\right) \quad F_{12 n}^{(m)}\left(k_{L n} r\right)\right]=-\rho_{n} \omega^{2}\left[J_{m}\left(k_{L n} r\right) \quad Y_{m}\left(k_{L n} r\right)\right]-\frac{2 \rho_{n} \omega c_{T n}^{2}}{c_{L n} r}\left[f_{m}^{(1)}\left(k_{L n} r\right) \quad f_{m}^{(2)}\left(k_{L n} r\right)\right]
$$

and

$$
\left[F_{23 n}^{(m)}\left(k_{T n} r\right) \quad F_{24 n}^{(m)}\left(k_{T n} r\right)\right]=\rho_{n} \omega^{2}\left[J_{m}\left(k_{T n} r\right) \quad Y_{m}\left(k_{T n} r\right)\right]+\frac{2 \rho_{n} \omega c_{T n}}{r}\left[f_{m}^{(1)}\left(k_{T n} r\right) \quad f_{m}^{(2)}\left(k_{T n} r\right)\right] .
$$

The functions $f_{m}^{(1)}, f_{m}^{(2)}, g_{m}^{(1)}$, and $g_{m}^{(2)}$ are combinations of Bessel functions given below:

$$
\begin{aligned}
& f_{m}^{(1)}(x)=J_{m}^{\prime}(x)-\frac{m^{2}}{x} J_{m}(x) \\
& f_{m}^{(2)}(x)=Y_{m}^{\prime}(x)-\frac{m^{2}}{x} Y_{m}(x) \\
& g_{m}^{(1)}(x)=J_{m}^{\prime}(x)-\frac{1}{x} J_{m}(x) \\
& g_{m}^{(2)}(x)=J_{m}^{\prime}(x)-\frac{1}{x} J_{m}(x)
\end{aligned}
$$


This completes the specification of the matrix $\mathbf{Z}^{(m)}$.

The last part of the solution is determining the forcing vector $\mathbf{b}^{(m)}$ for each mode. We will consider two cases, normal point load on the inside surface $r=R_{0}$, and normal point load on the outer surface $r=R_{N}$. In either case we can assume the load is applied at angular position $\theta=0$ with no loss of generality. The mathematical expression for the normal point load is

$$
\sigma_{r r}(R, \theta)=-\frac{1}{R} \delta(\theta)=-\frac{1}{2 \pi R} \sum_{m=0}^{\infty} \varepsilon_{m} \cos (m \theta),
$$

with $R=R_{0}$ for the inside surface, or $R=R_{N}$ for the outside surface. After substituting the Fourier series (5) into the expression for the normal stress in (2), then equating it with (16) above, we obtain the following for the forcing vectors $\mathbf{b}_{0}^{(m)}$ and $\mathbf{b}_{N}^{(m)}$ for the inside and outside surfaces:

$$
\mathbf{b}_{0}^{(m)}=-\frac{i^{m}}{2 \pi R_{0}}\left[\begin{array}{c}
1 \\
0 \\
\vdots \\
0
\end{array}\right] \quad, \quad \mathbf{b}_{N}^{(m)}=-\frac{i^{m}}{2 \pi R_{N}}\left[\begin{array}{c}
0 \\
\vdots \\
0 \\
1 \\
0
\end{array}\right]
$$

Once the coefficients for each mode are obtained by solving $\mathbf{Z}^{(m)} \cdot \mathbf{C}^{(m)}=\mathbf{b}^{(m)}$, we would like to calculate the normal displacement on the inner and outer surfaces. Using the series expansions (5) for the potentials and their relationships to the displacements (1) we obtain the following:

$$
\begin{aligned}
u_{0}(\theta)=u\left(R_{0}, \theta\right)=k_{L 0} \sum_{m=0}^{\infty} \varepsilon_{m}(-i)^{m}\{ & J_{m}^{\prime}\left(k_{L 0} R_{0}\right) A_{0}^{(m)}+Y_{m}^{\prime}\left(k_{L 0} R_{0}\right) B_{0}^{(m)} \\
& \left.+\frac{m}{k_{L 0} R_{0}}\left[J_{m}\left(k_{T 0} R_{0}\right) D_{0}^{(m)}+\frac{m}{R_{0}} Y_{m}\left(k_{T 0} R_{0}\right) E_{0}^{(m)}\right]\right\}, \\
u_{N}(\theta)=u\left(R_{N}, \theta\right)=k_{L N} \sum_{m=0}^{\infty} \varepsilon_{m}(-i)^{m}\{ & J_{m}^{\prime}\left(k_{L N} R_{N}\right) A_{N}^{(m)}+Y_{m}^{\prime}\left(k_{L N} R_{N}\right) B_{N}^{(m)} \\
+ & \left.\frac{m}{k_{L N} R_{N}}\left[J_{m}\left(k_{T N} R_{N}\right) D_{N}^{(m)}+\frac{m}{R_{N}} Y_{m}\left(k_{T N} R_{N}\right) E_{N}^{(m)}\right]\right\} .
\end{aligned}
$$

Suppose we sample the normal displacements at angular positions $\theta=\theta_{n}=2 \pi n / N$ for $n=1, \ldots, N-1$, then we can extract the $j$ th term $(j \leq N)$ of either (18) or (19) by evaluating the weighted sums

$$
u_{0 j}=\sum_{n=0}^{N-1} u_{0}\left(\theta_{n}\right) \cos \left(\frac{2 \pi j n}{N}\right)
$$

for the inner surface, and

$$
u_{N j}=\sum_{n=0}^{N-1} u_{N}\left(\theta_{n}\right) \cos \left(\frac{2 \pi j n}{N}\right)
$$

for the outer surface. These are the quantities are plotted in the text to determine the vibrational response in each example.

Finally, there are two special cases that can occur: a fluid layer $\left(\mu_{n}=0\right.$ for some $\left.n\right)$, and a solid core cylinder $\left(R_{0}=0\right)$. In a fluid the shear stress is zero, which eliminates the potential $\psi_{n}$, so that $D_{n}^{(m)}=E_{n}^{(m)}=0$ identically. In addition, the shear stress in adjacent layers must go to zero at the interfaces with the fluid layer $\left(\sigma_{r \theta}^{(n-1)}\left(R_{n-1}, \theta\right)=0\right.$ and $\left.\sigma_{r \theta}^{(n+1)}\left(R_{n}, \theta\right)=0\right)$. Similarly, there is no continuity condition for the tangential displacements $\left(v_{n-1}\right.$ and $\left.v_{n+1}\right)$ in adjacent layers. Thus we can eliminate the columns of 
$Z^{(m)}$ corresponding to $D_{n}^{(m)}$ and $E_{n}^{(m)}$, along with the rows of $Z^{(m)}$ containing the first and third rows of $\mathbf{S}_{n}^{(m)}$. This modification can be done for each fluid layer to determine the final form for $Z^{(m)}$.

If the cylinder has a solid core $\left(R_{0}=0\right)$, then the condition that the potentials must be finite at $r=0$ forces $B_{1}^{(m)}=E_{1}^{(m)}=0$. It also eliminates the free surface condition for the innermost layer. We can then

eliminate the first two rows of $Z^{(m)}$ along with the second and fourth columns which correspond to $B_{1}^{(m)}$ and $E_{1}^{(m)}$ to determine the final form for $Z^{(m)}$.

\section{Acknowledgements / Disclaimer}

This document was prepared as an account of work sponsored by an agency of the United States Government. Neither the United States Government nor the University of California nor any of their employees, makes any warranty, express or implied, or assumes any legal liability or responsibility for the accuracy, completeness, or usefulness of any information, apparatus, product, or process disclosed, or represents that its use would not infringe privately owned rights. Reference herein to any specific commercial product, process, or service by trade name, trademark, manufacturer, or otherwise, does not necessarily constitute or imply its endorsement, recommendation, or favoring by the United States Government or the University of California. The views and opinions of authors expressed herein do not necessarily state or reflect those of the United States Government or the University of California, and shall not be used for advertising or product endorsement purposes.

This work was performed under the auspices of the U. S. Department of Energy by the University of California, Lawrence Livermore National Laboratory under Contract No. W-7405-Eng-48. 


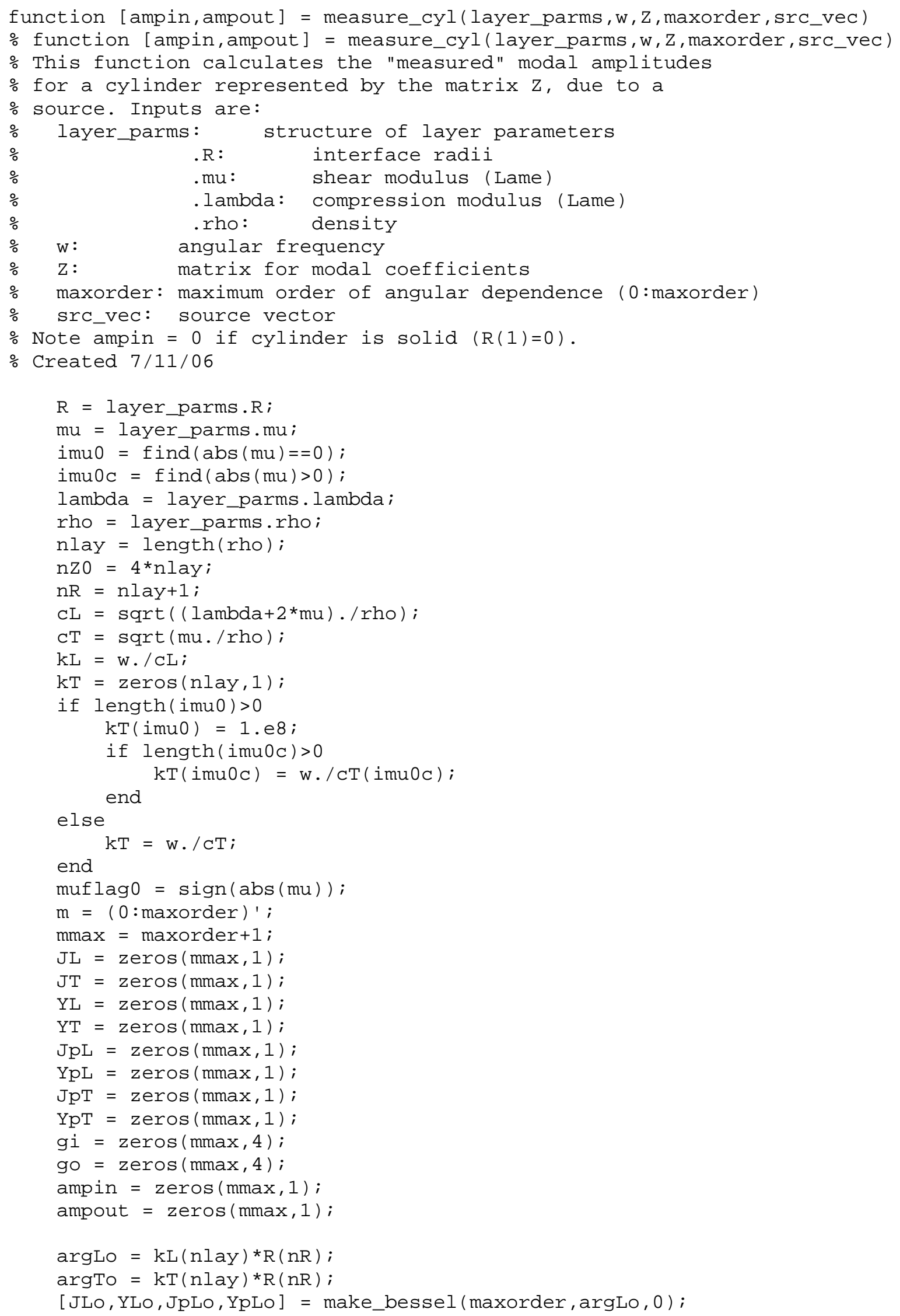


$[$ JTo, YTo, JpTo, YpTo $]=$ make_bessel $(\max o r d e r, \operatorname{argTo}, \odot)$;

go $(:, 1: 2)=\mathrm{kL}($ nlay $){ }^{*}$ [JpLo YpLo];

go $(:, 3)=\operatorname{muflag} \odot($ nlay $){ }^{*} \mathrm{~m} .{ }^{*} \mathrm{JTo} / \mathrm{R}(\mathrm{nR})$;

go $(:, 4)=\operatorname{muflag} \odot($ nlay $){ }^{*} m .{ }^{*} Y T o / R(n R)$;

if $\mathrm{R}(1)>0$

$\operatorname{argLi}=\mathrm{kL}(1){ }^{*} \mathrm{R}(1)$;

$\operatorname{argTi}=\mathrm{kT}(1) * \mathrm{R}(1)$;

$[\mathrm{JLi}, \mathrm{YLi}, \mathrm{JpLi}, \mathrm{YpLi}]=$ make_bessel $(\max$ order, $\operatorname{argLi}, \odot)$;

[JTi, YTi, JpTi, YpTi] = make_bessel $(\operatorname{maxorder}, \operatorname{argTi}, 0)$;

gi $(:, 1: 2)=\mathrm{kL}(1)^{*}[\mathrm{JpLi}$ YpLi];

$\mathrm{gi}(:, 3)=\operatorname{muflag} \Theta(1){ }^{*} \mathrm{~m} .{ }^{*} \mathrm{JTi} / \mathrm{R}(1)$;

end

$g i(:, 4)=\operatorname{muflag} \Theta(1){ }^{*} \mathrm{~m} \cdot{ }^{*} \mathrm{YTi} / \mathrm{R}(1)$;

for $j m=1: \operatorname{mmax}$

$\operatorname{tmp}=\operatorname{zeros}(n z \Theta, 1) ;$

$\mathrm{Z1}=\operatorname{squeeze}(\mathrm{Z}(:,:, j \mathrm{jm}))$;

$\operatorname{irZ1}=\operatorname{sign}(\operatorname{sum}(\operatorname{abs}(Z 1), 2))$;

itmp = find $($ irZ1)

$i c Z 1=\operatorname{sign}(\operatorname{sum}(\operatorname{abs}(Z 1), 1))$;

$\mathrm{nZ}=\operatorname{sum}(i r Z 1)$;

if $n Z<n Z \odot$

$\mathrm{Z} 2=\operatorname{zeros}(\mathrm{nZ}, \mathrm{nZ})$;

ind $=$ find $(i r Z 1 * i c Z 1>0)$;

$Z 2=Z 1$ (ind $Z$ );

$\mathrm{Z} 2=\operatorname{reshape}(\mathrm{Z2}, \mathrm{nZ}, \mathrm{nZ})$;

src_vec2 = src_vec (itmp);

$\%$

if $r$ cond $(Z 2)<1$. e-12

tmp2 = pinv $(Z 2) *$ src_vec2;

$\%$

$\%$

$\%$

$\%$

else

end

tmp2 = Z2\src_vec2;

else

$\operatorname{tmp}(i t m p)=\operatorname{tmp} 2$;

$\%$

$\%$

$\%$

if $r$ cond $(Z 1)<1 . e 12$

else

tmp $=\operatorname{pinv}(Z 1){ }^{*}$ src_vec;

tmp = Z1 \src_vec;

end

end

ampout $(j m)=\operatorname{squeeze}(g o(j m,:)){ }^{*} \operatorname{tmp}\left(\left(\left(4^{*}\right.\right.\right.$ nlay -3$):\left(4^{*}\right.$ nlay $\left.\left.)\right)\right)$;

if $R(1)>0$

end

$\operatorname{ampin}(j m)=\operatorname{squeeze}(g i(j m,:)){ }^{*} \operatorname{tmp}(1: 4) ;$

end

$\%$ end function 


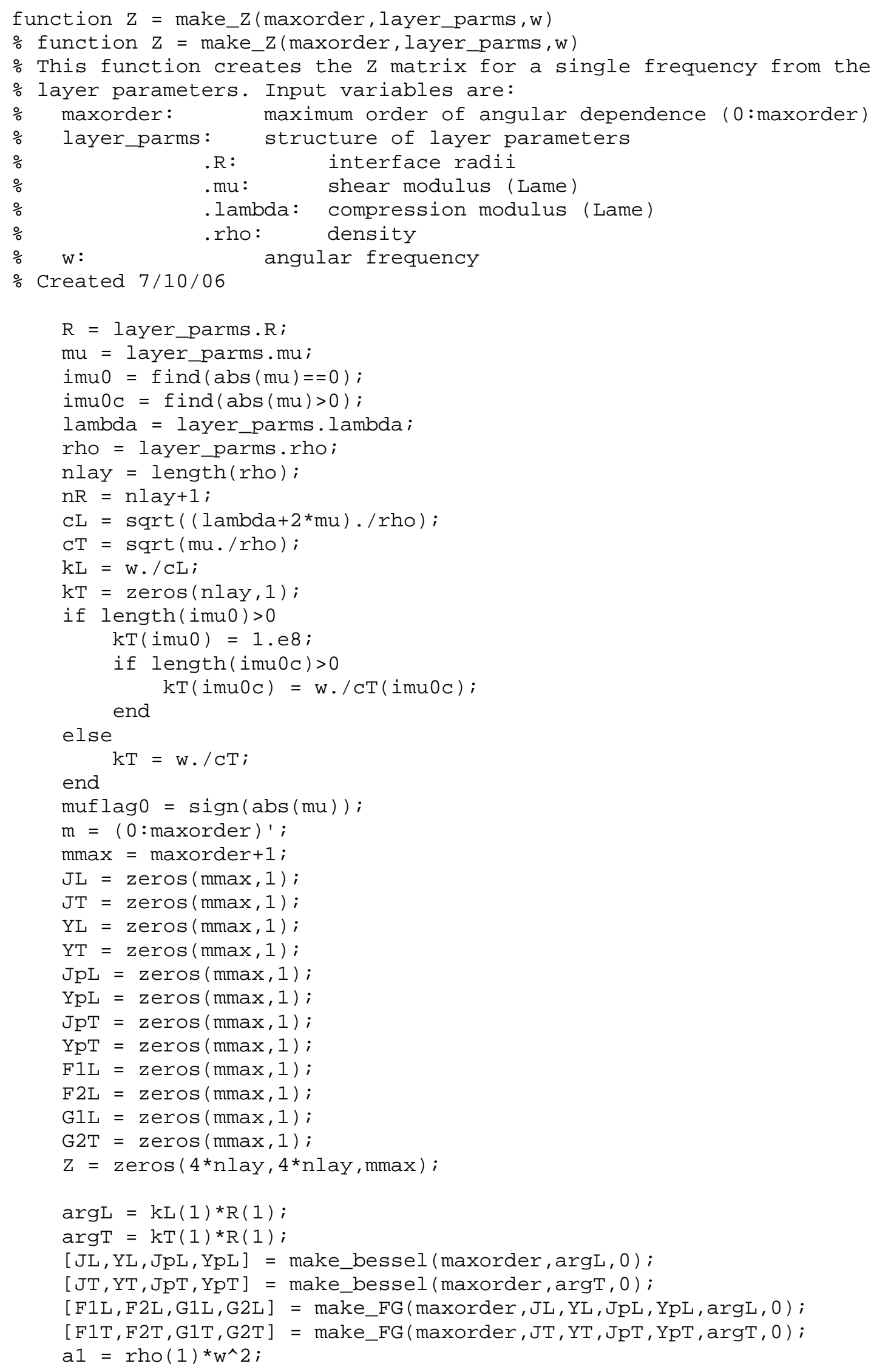




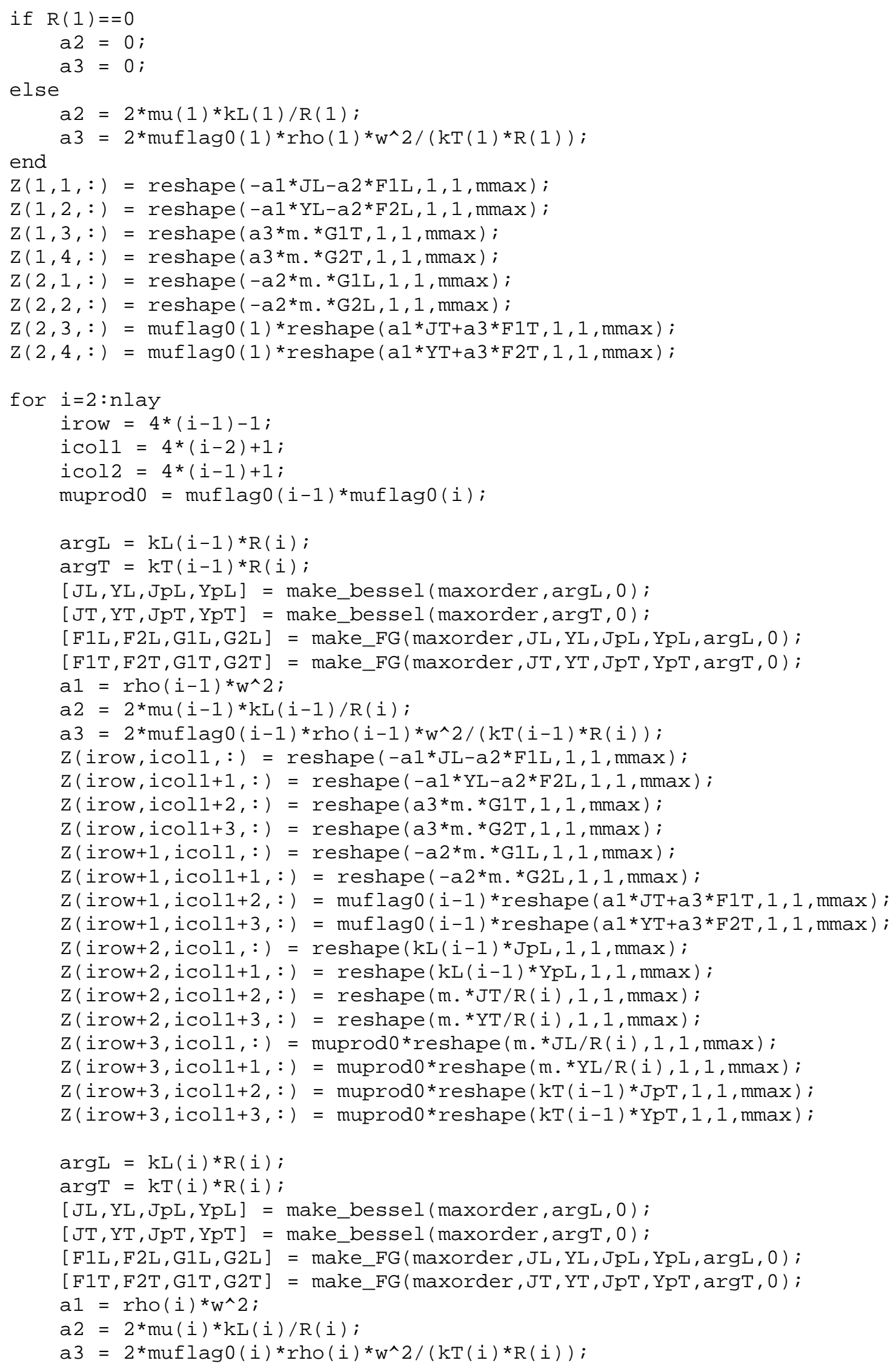




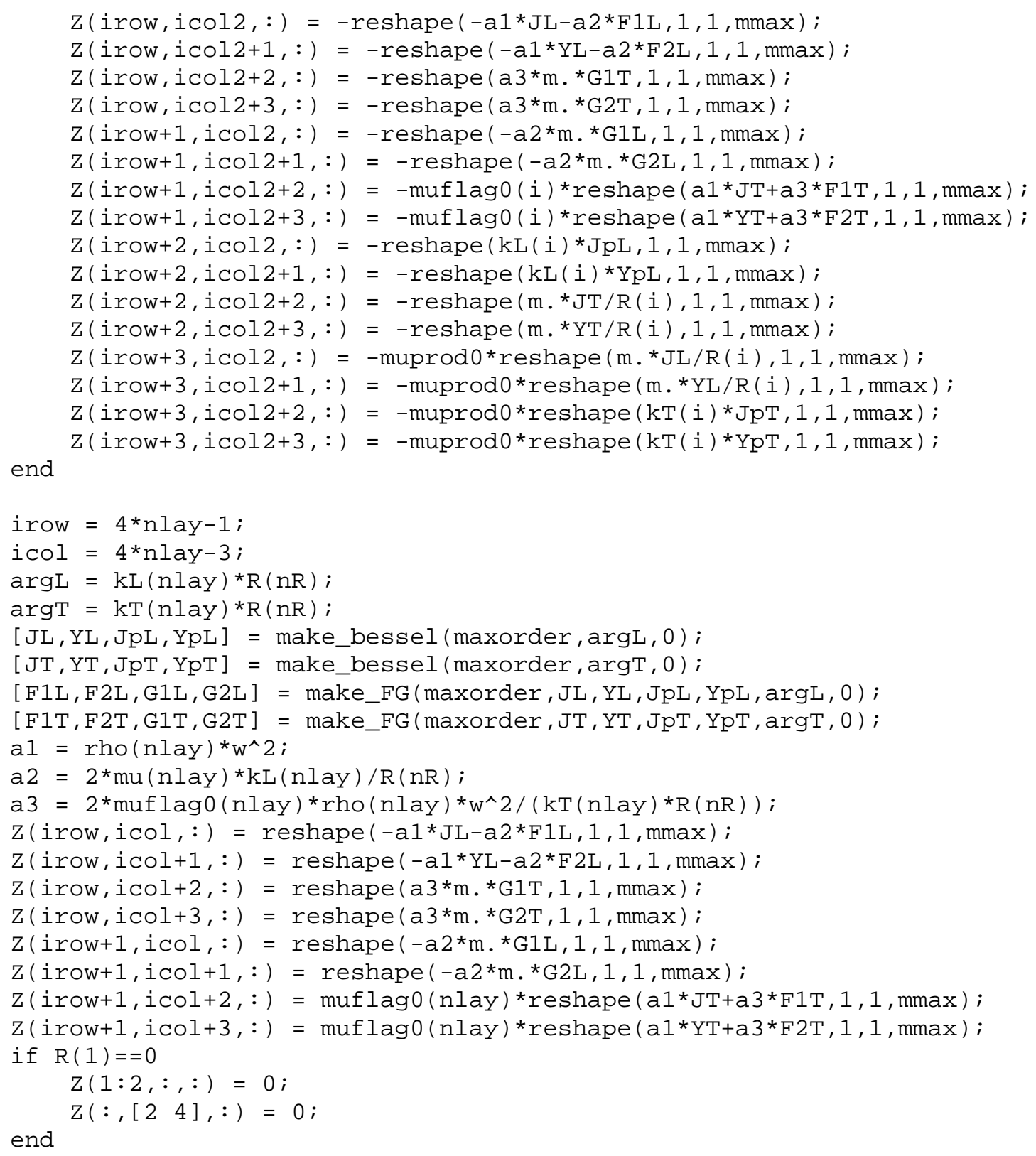

$\%$ end function 


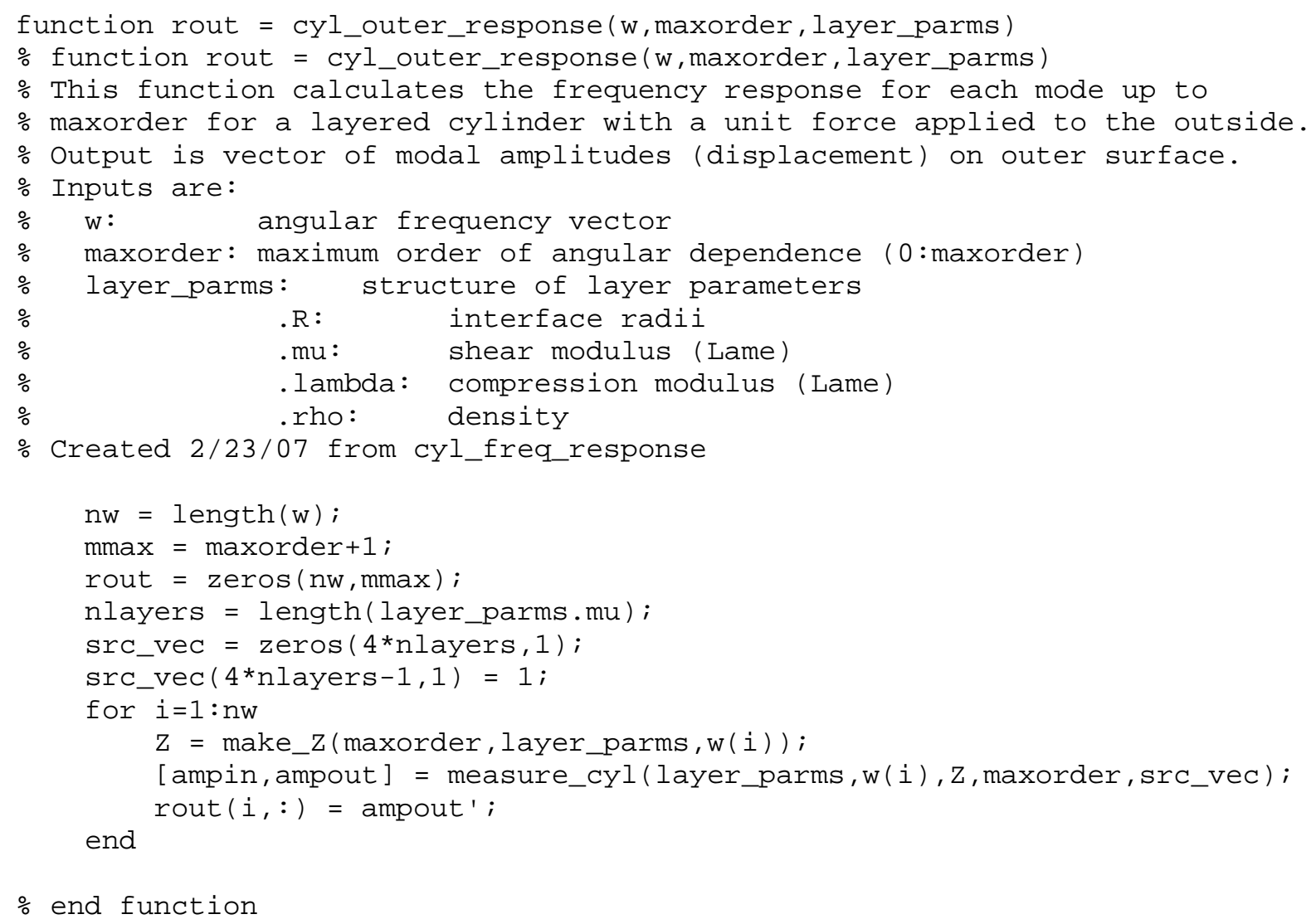


function $[F 1, F 2, G 1, G 2]=$ make_FG(maxorder, $J, Y, d J, d Y, x$, zeroflag)

$\%$ function $[F 1, F 2, G 1, G 2]=$ make_FG(maxorder, J, Y, dJ, dY, $x$, zeroflag)

$\%$ This function creates the F1, F2, G1, G2 functions from the

$\%$ Bessel functions and derivatives. If zeroflag is present set

$\% \mathrm{~F} 2$ and $\mathrm{G} 2$ to zero when $\mathrm{x}=0$.

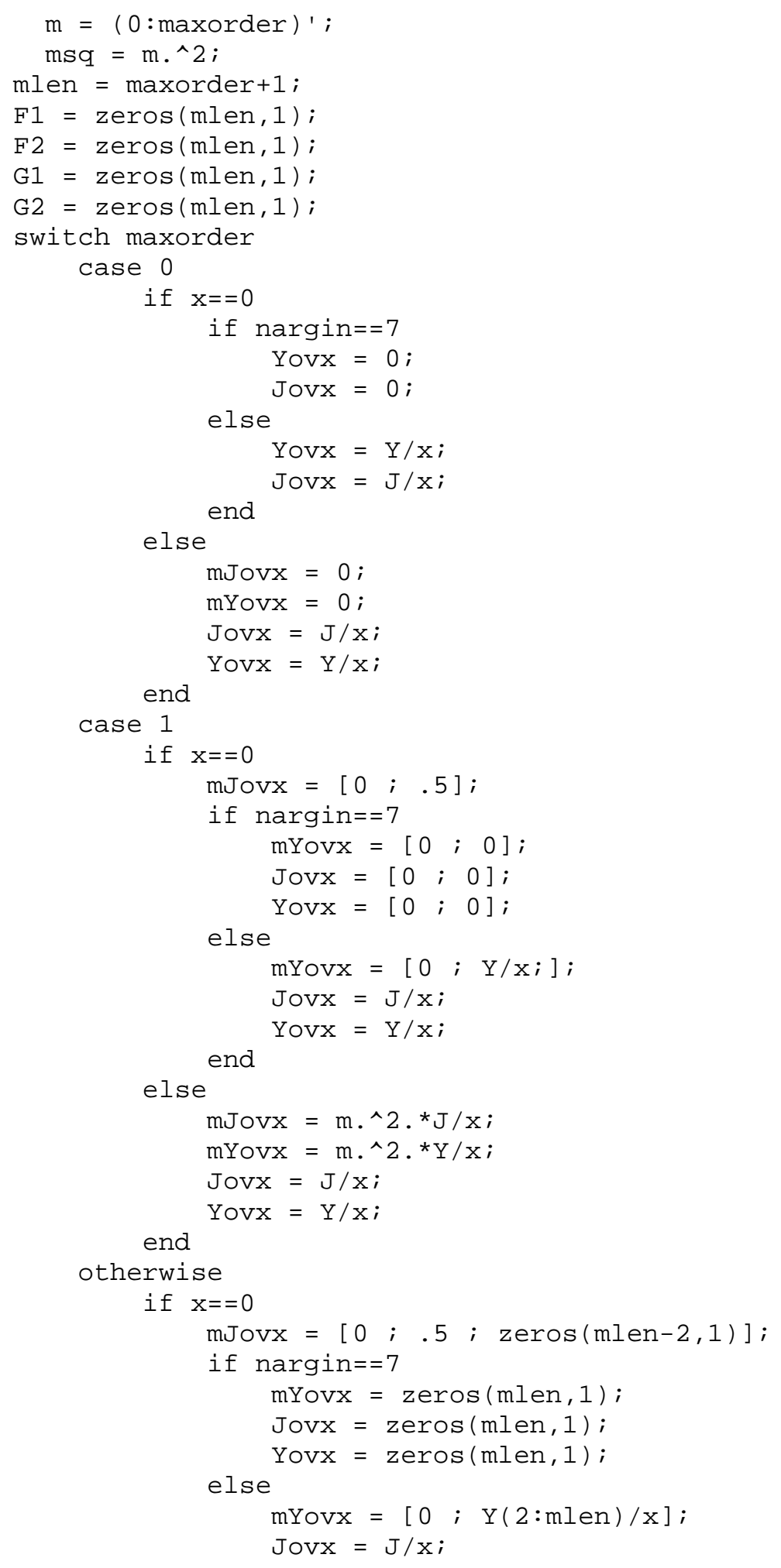




$$
\begin{aligned}
& \text { else }{ }^{\text {end }} \text { Yovx }=\mathrm{Y} / \mathrm{X} \text {; } \\
& \operatorname{mJovx}=\mathrm{m} \cdot \wedge 2 \cdot{ }^{*} \mathrm{~J} / \mathrm{x} ; \\
& \operatorname{mYovx}=\mathrm{m} \cdot \wedge 2 \cdot{ }^{*} \mathrm{Y} / \mathrm{x} \text {; } \\
& \operatorname{JovX}=\mathrm{J} / \mathrm{X} \text {; } \\
& \text { end } \\
& \text { YovX }=Y / X ; \\
& \text { end } \\
& \mathrm{F} 1=\mathrm{dJ}-\mathrm{mJovx} ; \\
& F 2=d Y-m Y o v x ; \\
& \mathrm{G} 1=\mathrm{dJ} \text {-Jovx; } \\
& \mathrm{G} 2=\mathrm{dY} \text {-YovX; } \\
& \% \quad F 1=d J-m . \wedge 2 .{ }^{*} / \mathrm{x} ; \\
& \% \quad \mathrm{~F} 2=\mathrm{dY}-\mathrm{m} . \wedge 2 \cdot{ }^{*} \mathrm{Y} / \mathrm{X} ; \\
& \% \quad \mathrm{G} 1=\mathrm{dJ}-\mathrm{J} / \mathrm{X} \text {; } \\
& \% \quad \mathrm{G} 2=\mathrm{d} Y-\mathrm{Y} / \mathrm{X} \text {; }
\end{aligned}
$$


function [Jn, Yn, dJn, dYn] = make_bessel(maxorder, arg, zeroflag)

$\%$ function [Jn, Yn, dJn, dYn] = make_bessel(maxorder, arg, zeroflag)

$\%$ This function calculates the Bessel function $\mathrm{J}, \mathrm{Y}$, and their

$\%$ derivatives (dJ, dY) for integer orders up to maxorder with a

$\%$ single argument. This is used to calculate acoustic fields for

$\%$ cylindrical objects. If zeroflag is present set $\mathrm{Y}$ and $\mathrm{dY}$ to

$\%$ zero when argument is zero.

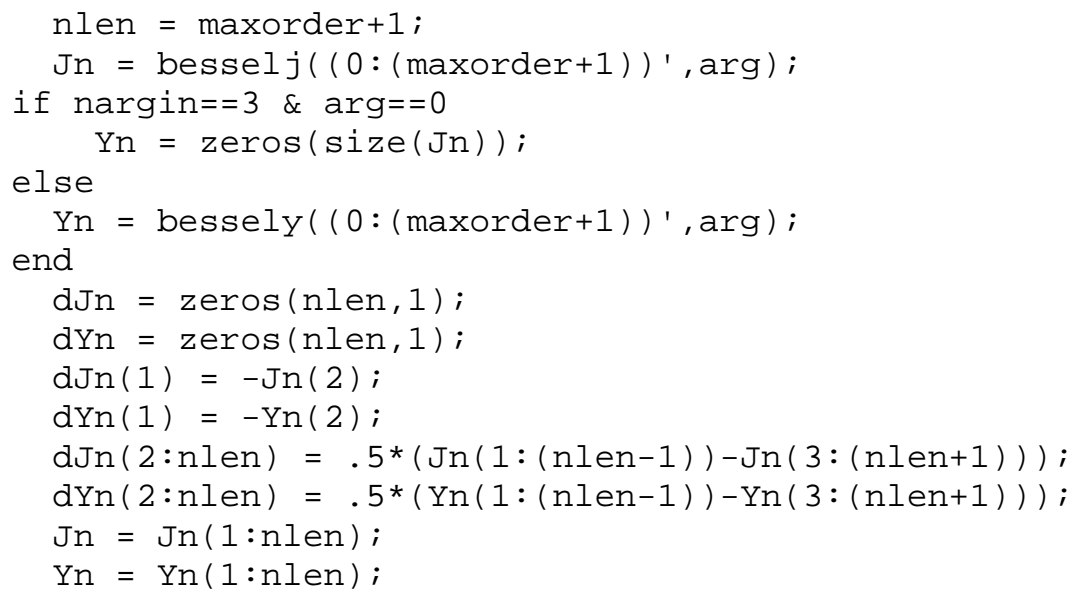

$\%$ end function 